\title{
On a Unification of Generalized Mittag-Leffler Function and Family of Bessel Functions
}

\author{
Jyotindra C. Prajapati ${ }^{1}$, Bhadresh I. Dave ${ }^{2}$, Bharti V. Nathwani ${ }^{2}$ \\ ${ }^{1}$ Department of Mathematical Sciences, Faculty of Applied Sciences, Charotar University of \\ Science and Technology, Changa, India \\ ${ }^{2}$ Department of Mathematics, Faculty of Science, The Maharaja Sayajirao University of Baroda, Vadodara, India \\ Email: jyotindra18@rediffmail.com, bidavemsu@yahoo.co.in, bharti.nathwani@yahoo.com
}

Received August 27, 2012; revised October 25, 2012; accepted November 7, 2012

\begin{abstract}
In the present work, a unification of certain functions of mathematical physics is proposed and its properties are studied. The proposed function unifies Lommel function, Struve function, the Bessel-Maitland function and its generalization, Dotsenko function, generalized Mittag-Leffler function etc. The properties include absolute and uniform convergence, differential recurrence relation, integral representations in the form of Euler-Beta transform, Mellin-Barnes transform, Laplace transform and Whittaker transform. The special cases namely the generalized hypergeometric function, generalized Laguerre polynomial, Fox H-function etc. are also obtained.
\end{abstract}

Keywords: Generalized Mittag-Leffler Function; Recurrence Relation; Wiman’s Function

\section{Introduction}

In the present work, we propose an extension of a generalization of the Mittag-Leffler function due to A. K. Shukla and J. C. Prajapati [1], defined as

$$
E_{\alpha, \beta}^{\gamma, q}(z)=\sum_{n=0}^{\infty} \frac{(\gamma)_{q n} z^{n}}{\Gamma(\alpha n+\beta) n !},
$$

where $\alpha, \beta, \gamma \in \mathbb{C} ; \operatorname{Re}(\alpha, \operatorname{Re}(\beta, \gamma))>0$ and $q \in(0,1) \cup \mathbb{N}$. This is an entire function of order $(\operatorname{Re} \alpha-q+1)^{-1}$ if $\operatorname{Re} \alpha>q-1$ and absolutely convergent in $\{|z|<R, R<1\}$ if $\operatorname{Re} \alpha=q-1$. In fact (1.1) contains the $E_{\alpha}(z)$-Mittag-Leffler function [2], $E_{\alpha, \beta}(z)$ the generalized Mittag-Leffler function [3] and the function $E_{\alpha, \beta}^{\gamma}(z)$ due to Prabhakar [4].

Gorenflo et al. [5], Saigo and Kilbas [6] studied several interesting properties of these functions.

Another generalization of Mittag-leffler function due to T. O. Salim [7], given by

$$
E_{\alpha, \beta}^{\gamma, \delta}(z)=\sum_{n=0}^{\infty} \frac{(\gamma)_{n} z^{n}}{\Gamma(\alpha n+\beta)(\delta)_{n}},
$$

where $z, \alpha, \beta, \gamma, \delta \in \mathbb{C}$ and

$$
\min \{\operatorname{Re}(\alpha, \beta, \delta)\}>0 .
$$

We state below the extended version in the form:

$$
\begin{aligned}
& E_{\alpha, \beta, \lambda, \mu, \rho, p}^{\gamma, \delta}(z ; s, r) \\
& =\sum_{n=0}^{\infty} \frac{\left[(\gamma)_{\delta n}\right]^{s} z^{(p n+\rho-1)}}{\Gamma(\alpha(p n+\rho-1)+\beta)\left[(\lambda)_{\mu n}\right]^{r}(\rho)_{p n}},
\end{aligned}
$$

where $\alpha, \beta, \gamma, \lambda, \rho \in \mathbb{C}, \operatorname{Re}(\alpha, \beta, \gamma, \lambda, \rho)>0, \delta, \mu, p>0$. The function defined by (1.2) reduces to the one in (1.1) and (1.1') if $\rho=1, p=1, r=0, s=1$ and $\delta=1$, $\rho=\delta, p=1, r=0, s=1$ respectively.

It is noteworthy that the function in (1.2), besides containing the generalizations of the Mittag-Leffler function, also includes certain functions belonging to the family of Bessel function. To see this, take $s=0, r=0, \rho=1$, $p=1, \alpha=1, \beta=v+1$, and replaced $z$ by $\frac{-z^{2}}{4}$ in (1.2), then we find the well known Bessel function [8]:

$$
J_{v}(z)=\sum_{n=0}^{\infty} \frac{(-1)^{n}}{n ! \Gamma(1+v+n)}\left(\frac{z}{2}\right)^{v+2 n} .
$$

When $s=0, r=0, \alpha=\mu, \beta=v+1$, and $z$ is replaced by $(-z)$ then we get the Bessel Maitland Function [8] given by $J_{v}^{\mu}(z)=\sum_{n=0}^{\infty} \frac{(-z)^{n}}{\Gamma(v+n \mu+1) n !}$. For $s=1$, $r=1, \quad \rho=1, \quad p=1, \quad \alpha=\mu, \quad \beta=v+\lambda+1, \quad \mu=1$, $\lambda=\lambda+1, \gamma=1, \delta=1$, and $z$ is replaced by $\frac{-z^{2}}{4}$, we 
obtain the Generalized Bessel Maitland function [8]:

$$
J_{v, \lambda}^{\mu}(z)=\sum_{n=0}^{\infty} \frac{(-1)^{n}}{\Gamma(v+n \mu+\lambda+1) \Gamma(n+\lambda+1)}\left(\frac{z}{2}\right)^{v+2 \lambda+2 n} .
$$

The Dotsenko Function [8]:

$$
\begin{aligned}
& { }_{2} R_{1}(a, b ; c, \omega ; \mu ; z) \\
& =\frac{\Gamma(c)}{\Gamma(a) \Gamma(b)} \sum_{n=0}^{\infty} \frac{\Gamma(a+n) \Gamma\left(b+n \frac{\omega}{\mu}\right)}{\Gamma\left(c+n \frac{\omega}{\mu}\right)} \frac{z^{n}}{n !}
\end{aligned}
$$

occurs by substituting $s=1, r=-1, \rho=1, \quad p=1$, $\alpha=c, \beta=\frac{\omega}{\mu}, \mu=\frac{\omega}{\mu}, \lambda=b, \gamma=a, \delta=1$ in (1.2).

The Lommel Function defined by [9]:

$$
\begin{aligned}
S_{\mu, v}(z)= & \frac{z^{\mu+1}}{(\mu-v+1)(\mu+v+1)^{1}} \\
& \cdot F_{2}\left(\begin{array}{cc}
1 & -\frac{z^{2}}{4} \\
1 / 2(\mu-v+3), & 1 / 2(\mu+v+3),
\end{array}\right)
\end{aligned}
$$

is the special case $s=1, r=1, \rho=1, p=1, \alpha=1$, $\beta=\frac{1}{2}(\mu-v+3), \quad \mu=1, \lambda=\frac{1}{2}(\mu+v+3), \quad \gamma=1, \quad \delta=1$, and $z$ is replaced by $\frac{-z^{2}}{4}$ of (1.2). On making substitutions $s=1, r=1, \rho=1, p=1, \alpha=1, \beta=3 / 2, \mu=1$, $\lambda=3 / 2+v, \quad \gamma=1, \delta=1$, and $z=\mp z^{2} / 4$ in (1.2), provides us respectively, the Struve Function $H_{v}(z)$ [9] given by

$$
H_{v}(z)=\frac{(z / 2)^{v+1}}{\Gamma(3 / 2) \Gamma(3 / 2+v)}{ }_{1} F_{2}\left(\begin{array}{cc}
1 & -\frac{z^{2}}{4} \\
3 / 2 & 3 / 2+v
\end{array}\right) .
$$

and the Modified Struve Function [9]:

$$
L_{v}(z)=\frac{(z / 2)^{v+1}}{\Gamma(3 / 2) \Gamma(3 / 2+v)}{ }_{1} F_{2}\left(\begin{array}{cc}
1 & -\frac{z^{2}}{4} \\
3 / 2 & 3 / 2+v
\end{array}\right) .
$$

In what follows, we shall use the following definitions and formulas. Euler (Beta) transform [10]:

$$
B\{f(z): a, b\}=\int_{0}^{1} z^{a-1}(1-z)^{b-1} f(z) \mathrm{d} z .
$$

Laplace transform [10]:

$$
\mathcal{L}\{f(z)\}=\int_{0}^{\infty} \mathrm{e}^{-s z} f(z) \mathrm{d} z .
$$

Mellin-Barnes transform [10]:

$$
M[f(z) ; z]=\int_{0}^{\infty} z^{s-1} f(z) \mathrm{d} z=f^{*}(s), \operatorname{Re}(s)>0,
$$

then

$$
f(z)=M^{-1}\left[f^{*}(s) ; x\right]=\frac{1}{2 \pi i} \int f^{*}(s) x^{-s} \mathrm{~d} s .
$$

Incomplete Gamma function [11]:

$$
\gamma(\alpha, z)=\int_{0}^{z} \mathrm{e}^{-t} t^{\alpha-1} \mathrm{~d} t, \operatorname{Re}(\alpha)>0 .
$$

The generalized hypergeometric function is denoted and defined by [11]

$$
{ }_{p} F_{q}\left[\begin{array}{l}
a_{1}, a_{2}, \cdots, a_{p} ; \\
b_{1}, b_{2}, \cdots, b_{q} ;
\end{array}\right]=\sum_{n=0}^{\infty} \frac{\left(a_{1}\right)_{n}\left(a_{2}\right)_{n} \cdots\left(a_{p}\right)_{n}}{\left(b_{1}\right)_{n}\left(b_{2}\right)_{n} \cdots\left(b_{q}\right)_{n}} \frac{z^{n}}{n !},
$$

where $b_{1}, b_{2}, \cdots, b_{q}$ are neither zero nor negative integers, and

$$
(\lambda)_{n}=\lambda(\lambda+1)(\lambda+2) \cdots(\lambda+n-1) .
$$

The series is convergent for 1) $|z|<\infty$ if $p \leq q, 2)$ $|z|<1$ if $p=q+1$.

Wright generalized hypergeometric function [12]:

$$
{ }_{p} \psi_{q}\left(\begin{array}{l}
\left(\alpha_{1}, A_{1}\right), \cdots,\left(\alpha_{p}, A_{p}\right) ; \\
\left(\beta_{1}, B_{1}\right), \cdots,\left(\beta_{q}, B_{q}\right) ;
\end{array}\right)=\sum_{n=0}^{\infty} \frac{\prod_{i=1}^{p} \Gamma\left(\alpha i+A_{i} n\right)}{\prod_{j=1}^{q} \Gamma\left(\beta j+B_{j} n\right)} \frac{z^{n}}{n !}
$$

Laguerre polynomial [12]:

$$
L_{n}^{(\alpha)}(x)=\frac{(1+\alpha)_{n}}{n !}{ }_{1} F_{1}\left[\begin{array}{c}
-n ; x \\
1+\alpha ;
\end{array}\right] .
$$

\section{Main Results}

In this section, we prove the following results for the function defined in (1.2).

Theorem 2.1. The series represented by the function $E_{\alpha, \beta, \lambda, \mu, \rho, p}^{\gamma, \delta}(z ; s, r)$ converges absolutely for $|z|<n^{1 / P(\operatorname{Re}(\mu) r+\Re(\alpha) p-\operatorname{Re}(\delta) s+p)}$.

Proof: Consider,

$$
\begin{aligned}
& E_{\alpha, \beta, \lambda, \mu, \rho, p}^{\gamma, \delta}(z ; s, r) \\
& =\sum_{n=0}^{\infty} \frac{\left[(\gamma)_{\delta n}\right]^{s} z^{p n+\rho-1}}{\Gamma(\alpha(p n+\rho-1)+\beta)\left[(\lambda)_{\mu n}\right]^{r}(\rho)_{p n}} .
\end{aligned}
$$

Take

$$
u_{n}=\frac{\left[(\gamma)_{\delta n}\right]^{s} z^{p n+\rho-1}}{\Gamma(\alpha(p n+\rho-1)+\beta)\left[(\lambda)_{\mu n}\right]^{r}(\rho)_{p n}}
$$

then 


$$
\begin{aligned}
& \left|\frac{u_{n}}{u_{n+1}}\right|=\left|\frac{\left[(\gamma)_{\delta n}\right]^{s} z^{p n+\rho-1}}{\Gamma(\alpha(p n+\rho-1)+\beta)\left[(\lambda)_{\mu n}\right]^{r}(\rho)_{p n}} \times \frac{\Gamma(\alpha(p(n+1)+\rho-1)+\beta)\left[(\lambda)_{\mu(n+1)}\right]^{r}(\rho)_{p(n+1)}}{\left[(\gamma)_{\delta(n+1)}\right]^{s} z^{p(n+1)+\rho-1}}\right| \\
& =\mid \frac{[\Gamma(\gamma+\delta n)]^{s}[\Gamma(\lambda)]^{r} \Gamma(\rho) z^{p n+\rho-1}}{[\Gamma(\gamma)]^{s} \Gamma(\alpha(p n+\rho-1)+\beta)[\Gamma(\lambda+\mu n)]^{r} \Gamma(\rho+p n)} \\
& \times \frac{[\Gamma(\gamma)]^{s} \Gamma(\alpha(p n+\rho-1)+\beta+\alpha p)[\Gamma(\lambda+\mu n+\mu)]^{r} \Gamma(\rho+p n+p)}{[\Gamma(\gamma+\delta n+\delta)]^{s}[\Gamma(\lambda)]^{r} \Gamma(\rho) z^{p(n+1)+\rho-1}} \mid \\
& =\left|\frac{[\Gamma(\gamma+\delta n)]^{s} \Gamma(\alpha n+\beta+\alpha p)[\Gamma(\lambda+\mu n+\mu)]^{r} \Gamma(\rho+p n+p)}{[\Gamma(\gamma+\delta n+\delta)]^{s} \Gamma(\alpha(p n+\rho-1)+\beta)[\Gamma(\lambda+\mu n)]^{r} \Gamma(\rho+p n) z^{p}}\right| \\
& =\mid \frac{[(\lambda+\mu n)(\lambda+\mu n+1)(\lambda+\mu n+2) \cdots(\lambda+\mu n+\mu-1)]^{r}}{[(\gamma+\delta n)(\gamma+\delta n+1)(\gamma+\delta n+2) \cdots(\gamma+\delta n+\delta-1)]^{s} z^{p}} \\
& \times(\alpha p n+\alpha \rho-\alpha+\beta)(\alpha p n+\alpha \rho-\alpha+\beta+1) \cdots(\alpha p n+\alpha \rho-\alpha+\beta+\alpha-1) \\
& \times(\rho+p n)(\rho+p n+1)(\rho+p n+2) \cdots(\rho+p n+p-1) \\
& =\mid \frac{n^{\mu r}\left[\left(\frac{\lambda}{n}+\mu\right)\left(\frac{\lambda+1}{n}+\mu\right)\left(\frac{\lambda+2}{n}+\mu\right) \cdots\left(\frac{\lambda+\mu-1}{n}+\mu\right)\right]^{r}}{n^{\delta s}\left[\left(\frac{\gamma}{n}+\delta\right)\left(\frac{\gamma+1}{n}+\delta\right)\left(\frac{\gamma+2}{n}+\delta\right) \cdots\left(\frac{\gamma+\delta-1}{n}+\delta\right)\right]^{s} z^{p}} \\
& \times n^{\alpha p}\left(\alpha p+\frac{\alpha \rho-\alpha+\beta}{n}\right)\left(\alpha p+\frac{\alpha \rho-\alpha+1}{n}\right) \cdots\left(\alpha p+\frac{\alpha \rho-\alpha+\beta+\alpha p-1}{n}\right) \\
& \times n^{p}\left(\frac{\rho}{n}+p\right)\left(\frac{\rho+1}{n}+p\right)\left(\frac{\rho+2}{n}+p\right) \cdots\left(\frac{\rho+p-1}{n}+p\right) .
\end{aligned}
$$

Thus,

$$
\lim _{n \rightarrow \infty}\left|\frac{u_{n}}{u_{n+1}}\right|=\lim _{n \rightarrow \infty}\left|n^{\mu r+\alpha p-\delta s+p} / z^{p}\right|
$$

Hence,

$$
\lim _{n \rightarrow \infty}\left|\frac{u_{n}}{u_{n+1}}\right|>1 \Rightarrow \lim _{n \rightarrow \infty}\left|n^{\mu r+\alpha p-\delta s+p} / z^{p}\right|>1 \Rightarrow|z|<n^{1 / p(\operatorname{Re}(\mu) r+\mathcal{H}(\alpha)-\operatorname{Re}(\delta) s+p)} .
$$

Theorem 2.2. For $\alpha, \beta, \gamma, \lambda, \rho \in \mathbb{C} ; \operatorname{Re}(\alpha)>0$ and $\delta, \mu, p>0$ the differential recurrence relation form:

$$
\beta E_{\alpha, \beta+1, \lambda, \mu, \rho, p}^{\gamma, \delta}(z ; s, r)+\alpha z \frac{\mathrm{d}}{\mathrm{d} z} E_{\alpha, \beta+1, \lambda, \mu, \rho, p}^{\gamma, \delta}(z ; s, r)=E_{\alpha, \beta, \lambda, \mu, \rho, p}^{\gamma, \delta}(z ; s, r) .
$$




\section{Proof.}

Consider,

$$
\begin{aligned}
& \beta E_{\alpha, \beta+1, \lambda, \mu, \rho, p}^{\gamma, \delta}(z ; s, r)+\alpha z \frac{\mathrm{d}}{\mathrm{d} z} E_{\alpha, \beta+1, \lambda, \mu, \rho, p}^{\gamma, \delta}(z ; s, r) \\
& =\beta \sum_{n=0}^{\infty} \frac{\left[(\gamma)_{\delta n}\right]^{s} z^{p n+\rho-1}}{\Gamma(\alpha(p n+\rho-1)+\beta+1)\left[(\lambda)_{\mu n}\right]^{r}(\rho)_{p n}}+\alpha z \frac{\mathrm{d}}{\mathrm{d} z} \sum_{n=0}^{\infty} \frac{\left[(\gamma)_{\delta n}\right]^{s}(z)^{p n+\rho-1}}{\Gamma(\alpha(p n+\rho-1)+\beta+1)\left[(\lambda)_{\mu n}\right]^{r}(\rho)_{p n}} \\
& =\beta \sum_{n=0}^{\infty} \frac{\left[(\gamma)_{\delta n}\right]^{s}(z)^{p n+\rho-1}}{\Gamma(\alpha(p n+\rho-1)+\beta+1)\left[(\lambda)_{\mu n}\right]^{r}(\rho)_{p n}}+\alpha z \sum_{n=0}^{\infty} \frac{\left[(\gamma)_{\delta n}\right]^{s}(p n+\rho-1)(z)^{p n+\rho-2}}{\Gamma(\alpha(p n+\rho-1)+\beta+1)\left[(\lambda)_{\mu n}\right]^{r}(\rho)_{p n}} \\
& =\sum_{n=0}^{\infty} \frac{\left[(\gamma)_{\delta n}\right]^{s}(z)^{p n+\rho-1}}{\Gamma(\alpha(p n+\rho-1)+\beta)\left[(\lambda)_{\mu n}\right]^{r}(\rho)_{p n}}=E_{\alpha, \beta, \lambda, \mu, \rho, p}^{\gamma}(z ; s, r)
\end{aligned}
$$

As the series given in (1.2) converges uniformly in any compact subset of $\mathbb{C}$, the use of term by term differentiation under the sign of summation leads us to the fol-

lowing theorem.

Theorem 2.3. If $m \in \mathbb{N}, \alpha, \beta, \gamma, \lambda, \rho \in \mathbb{C}$, $\operatorname{Re}(\alpha, \beta, \gamma, \lambda, \rho)>0$ and $\delta, \mu, p>0$ then

$$
\begin{gathered}
\left(\frac{\mathrm{d}}{\mathrm{d} z}\right)^{m} E_{\alpha, \beta, \lambda, \mu, \rho, p}^{\gamma, \delta}(z ; s, r)=\frac{z^{p m-m}\left[(\gamma)_{\delta m}\right]^{s} \Gamma(\rho)}{\left[(\lambda)_{\mu m}\right]^{r}\left[(\rho)_{p m-m}\right]} E_{\alpha, \beta+\alpha p m, \lambda+\mu m, \mu, \rho+p m-m, p}^{\gamma+\delta m, \delta}(z ; s, r), \\
\left(\frac{\mathrm{d}}{\mathrm{d} z}\right)^{m}\left[\mathrm{z}^{\beta-1} E_{\alpha, \beta, \lambda, \mu, \rho, p}^{\gamma, \delta}\left(\omega \mathrm{z}^{\alpha} ; s, r\right)\right]=z^{\beta-m-1} E_{\alpha, \beta-m, \lambda, \mu, \rho, p}^{\gamma, \delta}\left(\omega z^{\alpha} ; s, r\right), \text { if } \operatorname{Re}(\beta-m)>0 .
\end{gathered}
$$

Proof. Consider

$$
\begin{aligned}
& \left(\frac{\mathrm{d}}{\mathrm{d} z}\right)^{m} E_{\alpha, \beta, \lambda, \mu, r h o, p}^{\gamma, \delta}(z ; s, r)=\left(\frac{\mathrm{d}}{\mathrm{d} z}\right)^{m} \sum_{n=0}^{\infty} \frac{\left[(\gamma)_{\delta n}\right]^{s} z^{p n+\rho-1}}{[\Gamma(\alpha(p n+\rho-1)+\beta)]\left[(\lambda)_{\mu n}\right]^{r}\left[(\rho)_{p n}\right]} \\
& =\sum_{n=0}^{\infty} \frac{\left[(\gamma)_{\delta n}\right]^{s}}{[\Gamma(\alpha(p n+\rho-1)+\beta)]\left[(\lambda)_{\mu n}\right]^{r}\left[(\rho)_{p n}\right]}\left(\frac{\mathrm{d}}{\mathrm{d} z}\right)^{m}\left(z^{p n+\rho-1}\right) \\
& =\sum_{n=m}^{\infty} \frac{\left[(\gamma)_{\delta n}\right]^{s} \Gamma(\rho) z^{p n+\rho-m-1}}{[\Gamma(\alpha(p n+\rho-1)+\beta)]\left[(\lambda)_{\mu n}\right]^{r} \Gamma(p n+\rho-m)} \\
& =\sum_{n=0}^{\infty} \frac{\left[(\gamma)_{\delta(n+m)}\right]^{s} \Gamma(\rho) z^{p n+p m+\rho-m-1}}{[\Gamma(\alpha(p n+p m+\rho-1)+\beta)]\left[(\lambda)_{\mu(n+m)}\right]^{r} \Gamma(p n+p m+\rho-m)} \\
& =\sum_{n=0}^{\infty} \frac{\left[(\gamma)_{\delta m}\right]^{s}\left[(\gamma+\delta m)_{\delta n}\right]^{s} \Gamma(\rho) z^{p m-m}}{[\Gamma(\alpha(p n+\rho-1)+\beta+\alpha p m)]\left[(\lambda)_{\mu m}\right]^{r}\left[(\lambda+\mu m)_{\mu n}\right]^{r}} \frac{z^{p n+\rho-1}}{\Gamma(p n+p m+\rho-m)} \frac{\Gamma(\rho+p m-m)}{\Gamma(\rho+p m-m)} \\
& =\frac{z^{p m-m}\left[(\gamma)_{\delta m}\right]^{s} \Gamma(\rho)}{\left[(\lambda)_{\mu m}\right]^{r}\left[(\rho)_{p m-m}\right]} \times \sum_{n=0}^{\infty} \frac{\left[(\lambda+\delta m)_{\delta n}\right]^{s} z^{p n+\rho-1}}{\left[(\lambda+\mu)_{\mu n}\right]^{r}[\Gamma(\alpha(p n+\rho-1)+\beta+\alpha p m)](\rho+p m-m)_{p n}} \\
& =\frac{z^{p m-m}\left[(\gamma)_{\delta m}\right]^{s} \Gamma(\rho)}{\left[(\lambda)_{\mu m}\right]^{r}\left[(\rho)_{p m-m}\right]} E_{\alpha, \beta+\alpha p m, \lambda+\mu m, \mu, \rho+p m-m, p}^{\gamma+\delta m, \delta}(z ; s) .
\end{aligned}
$$


Now consider,

$$
\begin{aligned}
& \left(\frac{\mathrm{d}}{\mathrm{d} z}\right)^{m}\left[\mathrm{z}^{\beta-1} E_{\alpha, \beta, \lambda, \mu, \rho, p}^{\gamma, \delta}\left(\omega \mathrm{z}^{\alpha} ; s, r\right)\right]=\left(\frac{\mathrm{d}}{\mathrm{d} z}\right)^{m} \sum_{n=0}^{\infty} \frac{\left[(\gamma)_{\delta n}\right]^{s} z^{\alpha(p n+\rho-1)} z^{\beta-1} \omega^{p n+\rho-1}}{[\Gamma(\alpha(p n+\rho-1)+\beta)]\left[(\lambda)_{\mu n}\right]^{r}(\rho)_{p n}} \\
& =\sum_{n=0}^{\infty} \frac{\left[(\gamma)_{\delta n}\right]^{s} \omega^{p n+\rho-1}}{[\Gamma(\alpha(p n+\rho-1)+\beta)]\left[(\lambda)_{\mu n}\right]^{r}(\rho)_{p n}}\left(\frac{\mathrm{d}}{\mathrm{d} z}\right)^{m}\left(z^{\alpha(p n+\rho-1)+\beta-1}\right) \\
& =\sum_{n=0}^{\infty} \frac{\left[(\gamma)_{\delta n}\right]^{s}\left(\omega z^{\alpha}\right)^{(p n+\rho-1)} z^{\beta-m-1}}{[\Gamma(\alpha(p n+\rho-1)+\beta-m)]\left[(\lambda)_{\mu n}\right]^{r}(\rho)_{p n}}=z^{\beta-m-1} E_{\alpha, \beta-m, \lambda, \mu, \rho, p}^{\gamma, \delta}\left(\omega z^{\alpha} ; s, r\right)
\end{aligned}
$$

Next, taking $f(z)=E_{\alpha, \beta, \lambda, \mu}^{\gamma, \delta}\left(z u^{\alpha} ; s, r\right)$, in the Euler (Beta) transform (1.3), one finds the following
Theorem 2.4. If $\alpha, \beta, \gamma, \lambda, \rho, \sigma, \eta, v \in \mathbb{C}$,

$\operatorname{Re}(\alpha, \beta, \gamma, \lambda, \rho, \sigma, \eta, v)>0$ and $\delta, \mu, p>0$ then

$$
\begin{gathered}
\frac{1}{\Gamma(\eta)} \int_{0}^{1} u^{\beta-1}(1-u)^{\eta-1} E_{\alpha, \beta, \lambda, \mu, \rho, p}^{\gamma, \delta}\left(z u^{\alpha} ; s, r\right) \mathrm{d} u=E_{\alpha, \beta+\eta, \lambda, \mu, \rho, p}^{\gamma, \delta}(z ; s, r) \\
\frac{1}{\Gamma(\eta)} \int_{t}^{x}(x-t)^{\eta-1}(s-t)^{\beta-1} E_{\alpha, \beta, \lambda, \mu, \rho, p}^{\gamma, \delta}\left[v(s-t)^{\alpha} ; s, r\right] \mathrm{d} s=(x-t)^{\eta+\beta-1} E_{\alpha, \beta, \lambda, \mu, \rho, p}^{\gamma, \delta}\left[v(x-t)^{\alpha} ; s, r\right], \\
\int_{0}^{z} t^{\beta-1} E_{\alpha, \beta, \lambda, \mu, \rho, p}^{\gamma, \delta}\left[\omega t^{\alpha} ; s, r\right] \mathrm{d} t=z^{b} E_{\alpha, \beta+1, \lambda, \mu, \rho, p}^{\gamma, \delta}\left[\omega t^{\alpha} ; s, r\right], \\
\frac{1}{\Gamma(\sigma)} \int_{0}^{1} z^{\sigma-1}(1-z)^{\beta-1} E_{\alpha, \beta, \lambda, \mu, \rho, p}^{\gamma, \delta}\left(x(1-z)^{\alpha} ; s, r\right) \mathrm{d} z=E_{\alpha, \beta+\sigma, \lambda, \mu, \rho, p}^{\gamma, \delta}(x ; s, r) .
\end{gathered}
$$

\section{Proof.}

In (2.4.1),

$$
\begin{aligned}
\text { L.H.S. } & =\frac{1}{\Gamma(\eta)} \int_{0}^{1} u^{\beta-1}(1-u)^{\eta-1} E_{\alpha, \beta, \lambda, \mu, \rho, p}^{\gamma, \delta}\left(z u^{\alpha} s, r\right) \mathrm{d} u=\frac{1}{\Gamma(\eta)} \int_{0}^{1} u^{\beta-1}(1-u)^{\eta-1} \sum_{n=0}^{\infty} \frac{\left[(\gamma)_{\delta n}\right]^{s} u^{\alpha(p n+\rho-1)} z^{p n+\rho-1}}{\Gamma(\alpha n+\rho-1)+\beta)\left[(\lambda)_{\mu n}\right]^{r}(\rho)_{p n}} \mathrm{~d} u \\
& =\sum_{n=0}^{\infty} \frac{\left[(\gamma)_{\delta n}\right]^{s} z^{n}}{[\Gamma(\alpha(p n+\rho-1)+\beta)]\left[(\lambda)_{\mu n}\right]^{r}(\rho)_{p n} \Gamma(\eta)^{0}} \int^{1} u^{\alpha(p n+\rho-1)+\beta-1}(1-u)^{\eta-1} \mathrm{~d} u \\
& =\sum_{n=0}^{\infty} \frac{\left[(\gamma)_{\delta n}\right]^{s} z^{n}}{[\Gamma(\alpha(p n+\rho-1)+\beta)]\left[(\lambda)_{\mu n}\right]^{r}(\rho)_{p n} \Gamma(\eta)} \frac{\Gamma(\eta)[\Gamma(\alpha(p n+\rho-1)+\beta)]}{[(\alpha(p n+\rho-1)+\beta)+\eta]} \\
& =\sum_{n=0}^{\infty} \frac{\left[(\gamma)_{\delta n}\right]^{s} z^{p n+\rho-1}}{\Gamma(\alpha(p n+\rho-1)+\beta)\left[(\lambda)_{\mu n}\right]^{r}(\rho)_{p n} \Gamma(\eta)}=E_{\alpha, \beta+\eta, \lambda, \mu, \rho, p}^{\gamma, \delta}(z ; s, r)=R . H . S .
\end{aligned}
$$

Now, denoting the L.H.S. of (2.4.2) by $I$, we have

$$
\begin{aligned}
I & =\frac{1}{\Gamma(\eta)} \int_{t}^{x}(x-t)^{\eta-1}(s-t)^{\beta-1} E_{\alpha, \beta, \lambda, \mu, \rho, p}^{\gamma, \delta}\left[v(s-t)^{\alpha} ; s, r\right] \mathrm{d} s \\
& =\frac{1}{\Gamma(\eta)} \int_{t}^{x}(x-t)^{\eta-1}(s-t)^{\beta-1} \sum_{n=0}^{\infty} \frac{\left[(\gamma)_{\delta n}\right]^{s}(s-t)^{\alpha(p n+\rho-1)} v^{p n+\rho-1}}{\Gamma(\alpha n+\rho-1)+\beta)\left[(\lambda)_{\mu n}\right]^{r}(\rho)_{p n}} \mathrm{~d} s \\
& =\sum_{n=0}^{\infty} \frac{\left[(\gamma)_{\delta n}\right]^{s} v^{p n+\rho-1}}{\Gamma(\alpha(p n+\rho-1)+\beta)\left[(\lambda)_{\mu n}\right]^{r}(\rho)_{p n} \Gamma(\eta)^{t}(x-t)^{\eta-1}(s-t)^{\alpha(p n+\rho-1)+\beta-1} \mathrm{~d} s .}
\end{aligned}
$$


Here, introducing $u$ as a new variable of integration, by means of the relation

$$
u=\frac{s-t}{x-t},
$$

The further simplification gives,

$$
\begin{aligned}
I & =\sum_{n=0}^{\infty} \frac{\left[(\gamma)_{\delta n}\right]^{s} v^{p n+\rho-1}(x-t)^{\alpha(p n+\rho-1)+\beta+\eta-1}}{\Gamma(\alpha(p n+\rho-1)+\beta)\left[(\lambda)_{\mu n}\right]^{r}(\rho)_{p n} \Gamma(\eta)^{1}} \int_{0}^{(1-u)^{\eta-1}} u^{\alpha(p n+\rho-1)+\beta-1} \mathrm{~d} u \\
& =\sum_{n=0}^{\infty} \frac{\left[(\gamma)_{\delta n}\right]^{s} v^{p n+\rho-1}(x-t)^{\alpha(p n+\rho-1)+\beta+\eta-1}}{[\Gamma(\alpha(p n+\rho-1)+\beta)]\left[(\lambda)_{\mu n}\right]^{r}(\rho)_{p n} \Gamma(\eta)} \frac{\Gamma(\eta)[\Gamma(\alpha(p n+\rho-1)+\beta)]}{[\Gamma(\alpha(p n+\rho-1)+\beta+\eta)]} \\
& =\sum_{n=0}^{\infty} \frac{\left[(\gamma)_{\delta n}\right]^{s} v^{p n+\rho-1}(x-t)^{\alpha(p n+\rho-1)+\beta+\eta-1}}{[\Gamma(\alpha(p n+\rho-1)+\beta+\eta)]\left[(\lambda)_{\mu n}\right]^{r}(\rho)_{p n}} \\
& =(x-t)^{\eta+\beta-1} E_{\alpha, \beta+\eta, \lambda, \mu, \rho, p}^{\gamma, \delta}\left[v(x-t)^{\alpha} ; s, r\right] .
\end{aligned}
$$

as desired.

To prove (2.4.3) we begin with

$$
\begin{aligned}
& \int_{0}^{z} t^{\beta-1} E_{\alpha, \beta, \lambda, \mu, \rho, p}^{\gamma, \delta}\left(\omega t^{\alpha} ; s, r\right) \mathrm{d} t \\
& =\int_{0}^{z} t^{\beta-1} \sum_{n=0}^{\infty} \frac{\left[(\gamma)_{\delta n}\right]^{s} t^{\alpha(p n+\rho-1)} \omega^{p n+\rho-1}}{[\Gamma(\alpha(p n+\rho-1)+\beta)]\left[(\lambda)_{\mu n}\right]^{r}(\rho)_{p n}} \mathrm{~d} t \\
& =\sum_{n=0}^{\infty} \frac{\left[(\gamma)_{\delta n}\right]^{s} \omega^{n}}{[\Gamma(\alpha(p n+\rho-1)+\beta)]\left[(\lambda)_{\mu n}\right]^{r}(\rho)_{p n} \int_{0}^{z} t^{\alpha(p n+\rho-1)+\beta-1} \mathrm{~d} t} \\
& =\sum_{n=0}^{\infty} \frac{\left[(\gamma)_{\delta n}\right]^{s} z^{\alpha(p n+\rho-1)+\beta}(\omega)^{p n+\rho-1}}{[\Gamma(\alpha(p n+\rho-1)+\beta)]\left[(\lambda)_{\mu n}\right]^{r}(\rho)_{p n}(\alpha(p n+\rho-1)+\beta)} \\
& =\sum_{n=0}^{\infty} \frac{\left[(\gamma)_{\delta n}\right]^{s} z^{\alpha(p n+\rho-1)+\beta} \omega^{p n+\rho-1}}{[\Gamma(\alpha(p n+\rho-1)+\beta+1)]\left[(\lambda)_{\mu n}\right]^{r}(\rho)_{p n}}=z^{\beta} E_{\alpha, \beta+1, \lambda, \mu, \rho, p}^{\gamma, \delta}\left(\omega z^{\alpha} ; s, r\right) .
\end{aligned}
$$

Hence the result.

Now, consider

$$
\begin{aligned}
& \frac{1}{\Gamma(\sigma)} \int_{0}^{1} z^{\sigma-1}(1-z)^{\beta-1} E_{\alpha, \beta, \lambda, \mu, \rho, p}^{\gamma, \delta}\left(x(1-z)^{\alpha} ; s, r\right) \mathrm{d} z \\
& =\frac{1}{\Gamma(\sigma)} \int_{0}^{1} z^{\sigma-1}(1-z)^{\beta-1} \sum_{n=0}^{\infty} \frac{\left[(\gamma)_{\delta n}\right]^{s}(1-z)^{\alpha(p n+\rho-1)} x^{p n+\rho-1}}{[\Gamma(\alpha(p n+\rho-1)+\beta)]\left[(\lambda)_{\mu n}\right]^{r}(\rho)_{p n}} \mathrm{~d} z \\
& =\sum_{n=0}^{\infty} \frac{\left[(\gamma)_{\delta n}\right]^{s} x^{p n+\rho-1}}{[\Gamma(\alpha(p n+\rho-1)+\beta)]\left[(\lambda)_{\mu n}\right]^{r}(\rho)_{p n} \Gamma(\sigma)^{1}} \int_{0}^{1}(1-z)^{\alpha(p n+\rho-1)+\beta-1} z^{\sigma-1} \mathrm{~d} z \\
& =\sum_{n=0}^{\infty} \frac{\left[(\gamma)_{\delta n}\right]^{s} x^{p n+\rho-1}}{[\Gamma(\alpha(p n+\rho-1)+\beta)]\left[(\lambda)_{\mu n}\right]^{r}(\rho)_{p n} \Gamma(\sigma)} \frac{\Gamma(\sigma)[\Gamma(\alpha(p n+\rho-1)+\beta)]}{[\Gamma(\alpha(p n+\rho-1)+\beta)+\sigma]}
\end{aligned}
$$


simplification of above series yields (2.4.4).

\section{Mellin-Barnes Integral Representation of $E_{\alpha, \beta, \lambda, \mu}^{\gamma, \delta}(z ; s, r)$}

Theorem 3.1. Let $\alpha \in \mathbb{R}_{+} ; \beta, \gamma, \lambda, \rho, \in \mathbb{C}, \operatorname{Re}(\alpha, \beta, \gamma, \lambda, \rho)>0$ and $\delta, \mu>0, \quad p \in \mathbb{N}$. Then the function $E_{\alpha, \beta, \lambda, \mu, \rho, p}^{\gamma, \delta}(z ; s, r)$ is represented by the Mellin-Barnes integral as

$$
E_{\alpha, \beta, \lambda, \mu, \rho, p}^{\gamma, \delta}(z ; s, r)=\frac{[\Gamma(\lambda)]^{r} \Gamma(\rho) p z^{\rho-1}}{2 \pi i[\Gamma(\gamma)]^{s}} \int_{L} \frac{\Gamma(p S) \Gamma(1-p S)[\Gamma(\gamma-\delta S)]^{s}(-z)^{-p S}}{\Gamma(\beta+\alpha \rho-\alpha-\alpha p S)[\Gamma(\lambda-\mu S)]^{r} \Gamma(\rho-p S)} \mathrm{d} S,
$$

where $|\arg z|<\pi$; the contour of integration beginning at $-i \infty$ and ending at $+i \infty$, and indented to separate the poles of integrand at $S=-n$ for all $n \in \mathbb{N}_{0}$ (to the left) from those at $S=\frac{\gamma+n}{\delta}$ for all $n \in \mathbb{N}_{0}$ (to the right).

\section{Proof.}

We shall evaluate the integral on the R.H.S. of (3.1.1) as the sum of the residues at the poles $S=0,-1,-2, \cdots$ In fact, in view of the definition of residue, we have

$$
\begin{aligned}
I & =\frac{1}{2 \pi i} \int_{L} \frac{\Gamma(p S) \Gamma(1-p S)[\Gamma(\gamma-\delta S)]^{s}(-z)^{-p S}}{\Gamma(\beta+\alpha \rho-\alpha-\alpha p S)[\Gamma(\lambda-\mu S)]^{r} \Gamma(\rho-p S)} \mathrm{d} S \\
& =\sum_{n=0}^{\infty} S \operatorname{Res}\left[\frac{\Gamma(p S) \Gamma(1-p S)[\Gamma(\gamma-\delta S)]^{s}(-z)^{-p S}}{\Gamma(\beta+\alpha \rho-\alpha-\alpha p S)[\Gamma(\lambda-\mu S)]^{r} \Gamma(\rho-p S)}\right] \\
& =\sum_{n=0}^{\infty} \lim _{S \rightarrow-n} \frac{\pi(S+n)}{\sin \pi p S} \frac{[\Gamma(\gamma-\delta S)]^{s}(-z)^{-p S}}{\Gamma(\beta+\alpha \rho-\alpha-\alpha p S)[\Gamma(\lambda-\mu S)]^{r} \Gamma(\rho-p S)} \\
& =\sum_{n=0}^{\infty} \frac{(-1)^{p n}}{p} \frac{[\Gamma(\gamma-\delta S)]^{s}}{\Gamma(\beta+\alpha(p n+\rho-1))[\Gamma(\lambda+\mu n)]^{r} \Gamma(\rho+p n)}(-z)^{p n} .
\end{aligned}
$$

This gives,

$$
\begin{aligned}
I & =\frac{[\Gamma(\gamma)]^{s}}{[\Gamma(\lambda)]^{r} \Gamma(\rho) p z^{\rho-1}} \sum_{n=0}^{\infty} \frac{\left[(\gamma)_{\delta n}\right]^{s} z^{p n+\rho-1}}{\Gamma(\alpha(p n+\rho-1)+\beta)\left[(\lambda)_{\mu n}\right]^{r}(\rho)_{p n}} \\
& =\frac{[\Gamma(\gamma)]^{s}}{[\Gamma(\lambda)]^{r} \Gamma(\rho) p z^{\rho-1}} E_{\alpha, \beta, \lambda, \mu, \rho, p}^{\gamma, \delta}(z ; s, r) .
\end{aligned}
$$

\section{Integral Transforms of $E_{a, \beta, \lambda, \mu, \rho, p}^{\gamma, \delta}(z ; s, r)$}

In this section, we discussed some useful integral transforms like Euler transforms, Laplace transforms, Mellin transforms, Whittaker transforms,
For the convenience, we introduce the Notation:

$$
(\beta, \alpha)^{r}=\frac{[\Gamma(\beta+\alpha n)]^{r}}{[\Gamma(\beta)]^{r}}
$$

Theorem 4.1. (Euler(Beta) transforms)

$$
\begin{aligned}
& \int_{0}^{1} z^{a-1}(1-z)^{b-1} E_{\alpha, \beta, \lambda, \mu, \rho, p}^{\gamma, \delta}\left(x z^{\sigma} ; s, r\right) \mathrm{d} z \\
& =\frac{x^{\rho-1}[\Gamma(\lambda)]^{r} \Gamma(b) \Gamma(\rho)}{[\Gamma(\gamma)]^{s}}{ }_{s+2} \Psi_{r+3}\left[\begin{array}{cccc}
(\gamma, \delta)^{s}, & (\sigma \rho-\sigma+a, \sigma p), & (1,1) ; & x \\
(\alpha \rho-\alpha+\beta, \alpha p), & (\lambda, \mu)^{r}, & (\rho, p),(a+b, \sigma) ;
\end{array}\right],
\end{aligned}
$$

where $\alpha, \beta, \gamma, \lambda, \sigma, a, b \in \mathbb{C}, \operatorname{Re}(\alpha, \beta, \gamma, \lambda, \sigma, a, b)>0$ and $\delta, \mu p>0$. 


\section{Proof.}

$$
\begin{aligned}
& \int_{0}^{1} z^{a-1}(1-z)^{b-1} E_{\alpha, \beta, \lambda, \mu, \rho, p}^{\gamma, \delta}\left(x z^{\sigma} ; s, r\right) \mathrm{d} z=\int_{0}^{1} z^{a-1}(1-z)^{b-1} \sum_{n=0}^{\infty} \frac{\left[(\gamma)_{\delta n}\right]^{s} x^{p n+\rho-1} z^{\sigma(p n+\rho-1)}}{\Gamma(\alpha(p n+\rho-1)+\beta)\left[(\lambda)_{\mu n}\right]^{r}(\rho)_{p n}} \mathrm{~d} z \\
& =\sum_{n=0}^{\infty} \frac{\left[(\gamma)_{\delta n}\right]^{s} x^{p n+\rho-1}}{\Gamma(\alpha(p n+\rho-1)+\beta)\left[(\lambda)_{\mu n}\right]^{r}(\rho)_{p n}} \int_{0}^{1} z^{\sigma(p n+\rho-1)+a-1}(1-z)^{(b-1)} \mathrm{d} z \\
& =\sum_{n=0}^{\infty} \frac{\Gamma[(\gamma+\delta n)]^{s}[\Gamma(\lambda)]^{r} \Gamma(b) \Gamma(\sigma(p n+\rho-1)+a) x^{p n+\rho-1}}{\left[\Gamma(\alpha(p n+\rho-1)+\beta)[\Gamma(\lambda+\mu n)]^{r} \Gamma(\sigma(p n+\rho-1)+a+b)(\rho)_{p n}\right.} \\
& =\frac{x^{\rho-1}[\Gamma(\lambda)]^{r} \Gamma(b) \Gamma(\rho)}{[\Gamma(\lambda)]^{s}} \times{ }_{s+2} \Psi_{r+3}\left[\begin{array}{cccc}
(\gamma, \delta)^{s}, & (\sigma \rho-\sigma+a, \sigma p), & (1,1) ; & x \\
(\alpha \rho-\alpha+\beta, \alpha p), & (\lambda, \mu)^{r}, & (\rho, p),(a+b, \sigma) ;
\end{array}\right] .
\end{aligned}
$$

Theorem 4.2. (Laplace transforms)

$$
\begin{aligned}
& \int_{0}^{\infty} z^{a-1} \mathrm{e}^{-s z} E_{\alpha, \beta, \lambda, \mu, \rho, p}^{\gamma, \delta}\left(x z^{\sigma} ; s, r\right) \mathrm{d} z
\end{aligned}
$$

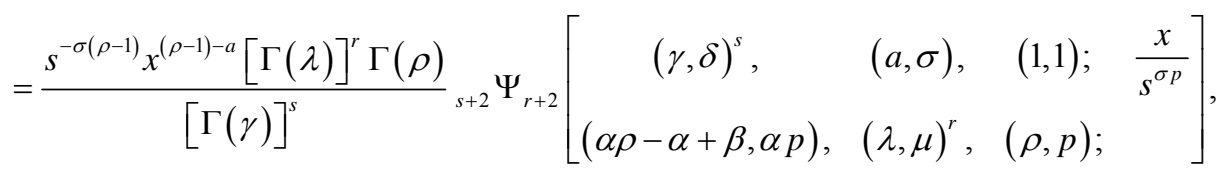

where $\alpha, \beta, \gamma, \lambda, \sigma, \rho, a, b \in \mathbb{C}, \operatorname{Re}(\alpha, \beta, \gamma, \lambda, \sigma, \rho, a, b)>0$ and $\operatorname{Re}(\alpha, \beta, \gamma, \lambda, \sigma, \rho, a, b)>0$.

Proof. We begin with

$$
\begin{aligned}
I & =\int_{0}^{\infty} z^{a-1} \mathrm{e}^{-s z} E_{\alpha, \beta, \lambda, \mu, \rho, p}^{\gamma, \delta}\left(x z^{\sigma} ; s, r\right) \mathrm{d} z=\int_{0}^{\infty} z^{a-1} \mathrm{e}^{-s z} \sum_{n=0}^{\infty} \frac{\left[(\gamma)_{\delta n}\right]^{s} x^{p n+\rho-1} z^{\sigma(p n+\rho-1)}}{\Gamma(\alpha(p n+\rho-1)+\beta)\left[(\lambda)_{\mu n}\right]^{r}(\rho)_{p n}} \mathrm{~d} z \\
& =\sum_{n=0}^{\infty} \frac{\left[(\gamma)_{\delta n}\right]^{s} x^{(p n+\rho-1)}}{\Gamma(\alpha(p n+\rho-1)+\beta)\left[(\lambda)_{\mu n}\right]^{r}(\rho)_{p n}} \int_{0}^{\infty} \mathrm{e}^{-s z} z^{\sigma(p n+\rho-1)+a-1} \mathrm{~d} z .
\end{aligned}
$$

On making substitution $t=s z$, we get

$$
\begin{aligned}
I & =\sum_{n=0}^{\infty} \frac{\left[(\gamma)_{\delta n}\right]^{s} x^{(p n+\rho-1)}}{\Gamma(\alpha(p n+\rho-1)+\beta)\left[(\lambda)_{\mu n}\right]^{r}(\rho)_{p n}} \int_{0}^{\infty} \mathrm{e}^{-t}(t / s)^{\sigma(p n+\rho-1)+a-1}(1 / s) \mathrm{d} t \\
& =\sum_{n=0}^{\infty} \frac{\left[(\gamma)_{\delta n}\right]^{s} x^{(p n+\rho-1)} s^{-\sigma(p n+\rho-1)-a}}{\Gamma(\alpha(p n+\rho-1)+\beta)\left[(\lambda)_{\mu n}\right]^{r}(\rho)_{p n}} \Gamma(\sigma(p n+\rho-1)+a) \\
& =\sum_{n=0}^{\infty} \frac{\Gamma[(\gamma+\delta n)]^{s}[\Gamma(\lambda)]^{r} x^{n} s^{-\sigma n-a} \Gamma(\sigma(p n+\rho-1)+a) \Gamma(\rho) \Gamma(n+1)}{[\Gamma(\gamma)]^{s} \Gamma(\alpha(p n+\rho-1)+\beta)[\Gamma(\lambda+\mu n)]^{r} \Gamma(\rho+p n) n !} \\
& =\frac{s^{-\sigma(\rho-1)} x^{\rho-1}[\Gamma(\lambda)]^{r}}{[\Gamma(\gamma)]^{s}} \Psi_{r+2}\left[\begin{array}{ccc}
(\gamma, \delta)^{s}, & (a, \sigma), \quad(1,1) ; & \frac{x}{s^{\sigma p}} \\
(\alpha \rho-\alpha+\beta, \alpha p), \quad(\lambda, \mu)^{r}, \quad(\rho, p),
\end{array}\right] .
\end{aligned}
$$

In proving the following theorem we use the integral formula involving the Whittaker function:

$$
\int_{0}^{\infty} t^{\nu-1} \mathrm{e}^{-t / 2} W_{\lambda, \mu}(t) \mathrm{d} t=\frac{\Gamma(1 / 2+\mu+v) \Gamma(1 / 2-\mu+v)}{\Gamma(1 / 2-\lambda+v)}, \operatorname{Re}(v \pm \mu)>-1 / 2
$$


Theorem 4.3. (Whittaker transforms)

$$
\begin{aligned}
& \int_{0}^{\infty} \mathrm{e}^{-q t / 2} t^{\psi-1} W_{\eta, \nu}(q t) E_{\alpha, \beta, \lambda, \mu, \rho, p}^{\gamma, \delta}\left(\omega t^{\sigma} ; s, r\right) \mathrm{d} t
\end{aligned}
$$

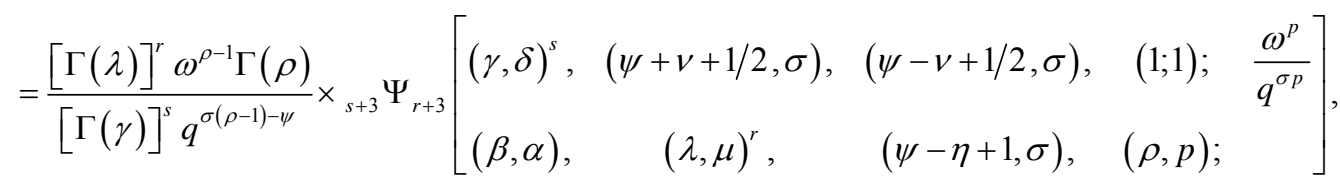

where $\alpha, \beta, \gamma, \lambda, \sigma, \rho, a, b \in \mathbb{C}, \operatorname{Re}(\alpha, \beta, \gamma, \lambda, \sigma, \rho, a, b)>0$ and $\delta, \mu, p>0$.

Proof. Let

$$
\begin{aligned}
I & =\int_{0}^{\infty} \mathrm{e}^{-q t / 2} t^{\psi-1} W_{\eta, \nu}(q t) E_{\alpha, \beta, \lambda, \mu, \rho, p}^{\gamma, \delta}\left(\omega t^{\sigma} ; s, r\right) \mathrm{d} t=\int_{0}^{\infty} \mathrm{e}^{-q t / 2} t^{\psi-1} W_{\eta, \nu}(q t) \sum_{n=0}^{\infty} \frac{\left[(\gamma)_{\delta n}\right]^{s} \omega^{p n+\rho-1} t^{\sigma(p n+\rho-1)}}{\Gamma(\alpha(p n+\rho-1)+\beta)\left[(\lambda)_{\mu n}\right]^{r}(\rho)_{p n}} \mathrm{~d} t \\
& =\sum_{n=0}^{\infty} \frac{\left[(\gamma)_{\delta n}\right]^{s} \omega^{p n+\rho-1}}{\Gamma(\alpha(p n+\rho-1)+\beta)\left[(\lambda)_{\mu n}\right]^{r}(\rho)_{p n}} \int_{0}^{\infty} \mathrm{e}^{-q t / 2} t^{\sigma(p n+\rho-1)+\psi-1} W_{\eta, \nu}(q t) \mathrm{d} t
\end{aligned}
$$

then using the substitution $\xi=q t$, we get

$$
\begin{aligned}
& I=\sum_{n=0}^{\infty} \frac{\left[(\gamma)_{\delta n}\right]^{s} \omega^{p n+\rho-1} q^{-\sigma(p n+\rho-1)-\psi}}{\Gamma(\alpha(p n+\rho-1)+\beta)\left[(\lambda)_{\mu n}\right]^{r}(\rho)_{p n}} \int_{0}^{\infty} \mathrm{e}^{-\xi / 2} \xi^{\sigma(p n+\rho-1)+\psi-1} W_{\eta, \nu}(\xi) \mathrm{d} \xi \\
& =\sum_{n=0}^{\infty} \frac{\Gamma[(\gamma+\delta n)]^{s}[\Gamma(\lambda)]^{r} \omega^{p n+\rho-1} q^{-\sigma(p n+\rho-1)-\psi}}{[\Gamma(\gamma)]^{s} \Gamma(\alpha(p n+\rho-1)+\beta)[\Gamma(\lambda+\mu n)]^{r}(\rho)_{p n}} \times \frac{\Gamma(\sigma(p n+\rho-1)+\psi+v+1 / 2) \Gamma(\sigma n+\psi-v+1 / 2)}{\Gamma(\sigma(p n+\rho-1)+\psi-\eta+1)}
\end{aligned}
$$

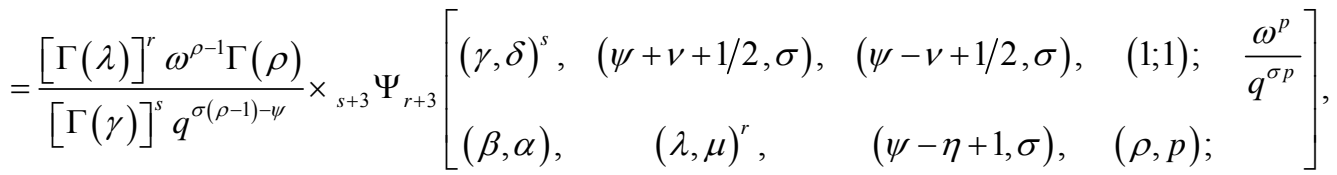

Theorem 4.4. (Mellin transforms)

$$
\int_{0}^{\infty} t^{p S-1} E_{\alpha, \beta, \lambda, \mu, \rho, p}^{\gamma, \delta}(-\omega t ; s, r) \mathrm{d} t=\frac{\Gamma(p S) \Gamma(1-p S)[\Gamma(\gamma-\delta S)]^{s}}{\omega^{p S} \Gamma(\beta+\alpha \rho-\alpha-\alpha p S)[\Gamma(\lambda-\mu S)]^{r} \Gamma(\rho-p S)}
$$

where $\alpha, \beta, \gamma, \lambda, \rho, S \in \mathbb{C}, \operatorname{Re}(\alpha, \beta, \gamma, \lambda, \rho, S)>0 \quad \delta, \mu, p>0$.

Proof. Putting $z=-\omega t$ in (3.1.1), we get

$$
\begin{aligned}
E_{\alpha, \beta, \lambda, \mu, \rho, p}^{\gamma, \delta}(z ; s, r) & =\frac{[\Gamma(\lambda)]^{r} \Gamma(\rho) p z^{\rho-1}}{2 \pi i[\Gamma(\gamma)]^{s}} \int_{L} \frac{\Gamma(p S) \Gamma(1-p S)[\Gamma(\gamma-\delta S)]^{s}(-\omega t)^{-p S}}{\Gamma(\beta+\alpha \rho-\alpha-\alpha p S)[\Gamma(\lambda-\mu S)]^{r} \Gamma(\rho-p S)} \mathrm{d} S \\
& =\frac{[\Gamma(\lambda)]^{r} \Gamma(\rho) p z^{\rho-1}}{2 \pi i[\Gamma(\lambda)]^{s}} \int_{L} f^{*}(S) t^{-p S} \mathrm{~d} S,
\end{aligned}
$$

in which

$$
f^{*}(S)=\frac{\Gamma(p S) \Gamma(1-p S)[\Gamma(\gamma-\delta S)]^{s}}{\omega^{p S} \Gamma(\beta+\alpha \rho-\alpha-\alpha p S)[\Gamma(\lambda-\mu S)]^{r} \Gamma(\rho-p S)}
$$

using (1.5) and (1.6) in (4.4.2), immediately leads us to (4.4.1). 


\section{Generalized Hypergeometric Function Representation of $E_{a, \beta, \lambda, \mu, \rho, p}^{\gamma, \delta}(z ; s, r)$}

Taking $\alpha=a, \delta=l, \mu=m$ in (1.2), we get

$$
\begin{aligned}
& E_{k, \beta, \lambda, m, \rho, p}^{\gamma, l}(z ; s, r)=\sum_{n=0}^{\infty} \frac{\left[(\gamma)_{\mathrm{ln}}\right]^{s} z^{p n+\rho-1}}{\Gamma(a(p n+\rho-1)+\beta)\left[(\lambda)_{m n}\right]^{r}(\rho)_{p n}}=\frac{1}{\Gamma(\beta+a \rho-a)} \sum_{n=0}^{\infty} \frac{\left[(\gamma)_{\mathrm{ln}}\right]^{s} z^{p n+\rho-1}(1)_{n}}{(\beta+a \rho-a)_{a p n}\left[(\lambda)_{\mu n}\right]^{r}(\rho)_{p n} n !} \\
& =\frac{z^{\rho-1}}{\Gamma(\beta+a \rho-a)} \sum_{n=0}^{\infty} \frac{\left[\prod_{i=1}^{l}\left(\frac{\gamma+i-1}{l}\right)_{n}\right]^{s}\left[\prod_{k=1}^{m}\left(\frac{\lambda+k-1}{m}\right)_{n}\right]^{-r}(1)_{n}}{\prod_{j=1}^{a}\left(\frac{\beta+a \rho-a+j-1}{a p}\right)_{n}^{p}\left(\frac{\rho+h-1}{p}\right)_{n}} \times \frac{l^{l s n} z^{p n} p^{p n}(a p)^{a p n}}{n !} \\
& =\frac{z^{\rho-1}}{\Gamma(\beta+a \rho-a)} \times{ }_{{ }^{5}} F_{a p+m^{r}+p}\left[\begin{array}{ccc}
\Delta(l, \gamma)^{s}, & 1 ; & \frac{l^{l s} z^{p}}{m^{m r} p^{p}(a p)^{a p}} \\
\Delta(a p, \beta+a \rho-a), & \Delta(m, \lambda)^{r}, \Delta(p, \rho) ;
\end{array}\right]
\end{aligned}
$$

where $\Delta(n ; \alpha)$ is a $n$-tuple $\frac{\alpha}{n}, \frac{\alpha+1}{n}, \cdots, \frac{\alpha+n-1}{n}$.

\section{Relationship with Some Known Special Functions (Generalized Laguerre Polynomial, Fox H-Function, Wright Hypergeometric Function)}

\subsection{Relationship with Generalized Laguerre Polynomials}

Putting $\alpha=k, \beta=v+1, \gamma=-m, r=0, s=1, \rho=1, \quad p=1$ and replacing $\delta$ by $q \in \mathbb{N}$ and $z$ by $z^{k}$ in (1.2), we get

$$
\begin{aligned}
E_{k, v+1, \lambda, \mu, 1,1}^{-m, q}(z ; 1,0) & =\sum_{n=0}^{\left[\frac{m}{q}\right]} \frac{\left[(-m)_{q n}\right]}{\Gamma(k n+v+1)} \frac{z^{k n}}{n !}=\sum_{n=0}^{\left[\frac{m}{q}\right]} \frac{(-1)^{q n} m !}{(m-q n) !} \frac{1}{\Gamma(k n+v+1)} \frac{z^{k n}}{n !} \\
& =\frac{\Gamma(m+1)}{\Gamma(k m+v+1)} \sum_{n=0}^{\left[\frac{m}{q}\right]} \frac{(-1)^{q n}}{(m-q n) !} \frac{\Gamma(k m+v+1)}{\Gamma(k n+v+1)} \frac{z^{k n}}{n !}=\frac{\Gamma(m+1)}{\Gamma(k m+v+1)} Z_{\left[\frac{m}{q}\right]}^{(v)}(z, k)
\end{aligned}
$$

where $Z_{\left[\frac{m}{q}\right]}^{(v)}(z, k)$ is polynomial of degree $\left[\frac{m}{q}\right]$ in $z^{k}$.

In particular, $Z_{m}^{(v)}(z, 1)=L_{m}^{(v)}(z)$, so that

$$
E_{k, v+1, \lambda, \mu, 1,1}^{-m, 1}(z ; 1,0)=\frac{\Gamma(m+1)}{\Gamma(k m+v+1)} L_{m}^{(v)}(z)
$$

\subsection{Relationship with Fox H-Function}

From (3.1.1), we have

$$
\begin{aligned}
& E_{\alpha, \beta, \lambda, \mu, \rho, p}^{\gamma, \delta}(z ; s, r)=\frac{[\Gamma(\lambda)]^{r} \Gamma(\rho)}{2 \pi i[\Gamma(\gamma)]^{s}} \times \int_{L} \frac{\Gamma(S)[\Gamma(\gamma-\delta S)]^{s}}{\Gamma(\beta+\alpha \rho-\alpha-\alpha p S)[\Gamma(\lambda-\mu S)]^{r} \Gamma(\rho-p S)}(-z)^{-s} \mathrm{~d} S \\
& =\frac{[\Gamma(\lambda)]^{r} \Gamma(\rho)}{[\Gamma(\gamma)]^{s}} \times H_{s, r+3}^{1, s}\left(-z \mid \begin{array}{cc}
{[(1-\gamma, \delta)]^{s} ;} \\
(0,1), & (1-\beta-\alpha \rho+\alpha, \alpha p), \quad[(1-\lambda, \mu)]^{r}, \quad(1-\rho, p) ;
\end{array}\right) .
\end{aligned}
$$




\subsection{Relationship with Wright Function}

If $\alpha, \beta, \gamma, \lambda, \rho \in \mathbb{C}, \operatorname{Re}(\alpha, \beta, \gamma, \lambda, \rho)>0, \delta, \mu, p>0 \quad$ (1.2) can be written as

$$
E_{\alpha, \beta, \lambda, \mu, \rho, p}^{\gamma, \delta}(z ; s, r)=\frac{[\Gamma(\lambda)]^{r} \Gamma(\rho)}{[\Gamma(\gamma)]^{s}} \sum_{n=0}^{\infty} \frac{[\Gamma(\gamma+\delta n)]^{s} z^{p n+\rho-1}}{\Gamma(\alpha(p n+\rho-1)+\beta)[(\lambda+\mu n)]^{r} \Gamma(\rho+p n)},
$$

from (1.9) for (6.3.1), we get

$$
=\frac{[\Gamma(\lambda)]^{r} \Gamma(\rho) z^{\rho-1}}{[\Gamma(\gamma)]^{s}}{ }_{s+1} \Psi_{r+2}\left[\begin{array}{ccc}
{[(\gamma, \delta)]^{s},} & (1,1) ; & z^{p} \\
(\beta+\alpha \rho-\alpha, \alpha p), & {[(\lambda, \mu)]^{r},} & (\rho, p) ;
\end{array}\right]
$$

\section{Summary}

In Section 1, an extended version of Mittag-Leffler function of 10 indices established as an Equation (1.2) including with some necessary information of Bessel function, some well-known integral transforms and generalized hypergeometric functions with their family. Results obtained in Sections 2 to 6 are interesting generalizations of (Shukla and Prajapati [1]) and stimulate the scope of further research in the field of generalization MittagLeffler function.

\section{Acknowledgements}

This paper dedicated to our beloved great Mathematician Gösta Mittag-Leffler. The authors would like to thank the reviewers for their valuable suggestions to improve the quality of paper.

\section{REFERENCES}

[1] A. K. Shukla and J. C. Prajapti, "On a Generlization of Mittag-Leffler Functions and Its Properties," Journal of Mathematical Analysis and Applications, Vol. 336, No. 2, 2007, pp. 797-811. doi:10.1016/j.jmaa.2007.03.018

[2] G. Mittag-Leffler, "Sur la Nouvelle Fonction $E_{\alpha}(x)$," Comptes Rendus de l'Academie des Sciences Paris, Vol. 137, 1903, pp. 554-558.

[3] A. Wiman, "Über die Nullstellen der Funktionen $E_{\alpha}(x)$,"
Acta Mathematica, Vol. 29, No. 1, 1905, pp. 217-234. doi:10.1007/BF02403204

[4] T. R. Prabhakar, "A Singular Equation with a Generalized Mittag-Leffler Function in the Kernel," Yokohama Mathematical Journal, Vol. 19, 1971, pp. 7-15.

[5] R. Gorenflo, A. A. Kilbas and S. V. Rogosin, "On the Generalized Mittag-Leffler Type Function," Integral Transforms and Special Functions, Vol. 7, No. 3-4, 1998, pp. 215-224. doi:10.1080/10652469808819200

[6] M. Saigo and A. A. Kilbas, "On Mittag Leffler Type Function and Applications," Integral Transforms and Special Functions, Vol. 7, No. 1-2, 1998, pp. 97-112. doi:10.1080/10652469808819189

[7] T. O. Salim, "Some Properties Relating to the Generalized Mittag-Leffler Function," Advances in Applied Mathematical Analysis, Vol. 4, No. 1, 2009, pp. 21-30.

[8] H. J. Haubold, A. M. Mathai and R. K. Saxena, "The H-Function: Theory and Applications," Publication No. 37 of Centre for Mathematical Sciences, Pala Campus, 2008.

[9] Y. L. Luke, "The Special Functions and their approximations," Academic Press, New York, London, 1969.

[10] I. N. Sneddon, “The Use of Integral Transforms," McGrawHill Book Company, New York, 1972.

[11] E. D. Rainville, "Special Functions," Macmillan Co., New York, 1960.

[12] H. M. Srivastava and H. L. Manocha, "A Treatise on Generating Functions,” Ellis Horwood Ltd., Chichester, 1984. 